\title{
Esquemas de Modulação Codificada Espectralmente Assimétricos
}

\author{
Antonio Cláudio F. Pessoa e Dalton Soares Arantes
}

Neste trabalho, é apresentada uma classe de esquemas de modulaçăo codificada controlados por códigos de bloco binários que utilizam constelaçőes regulares combinando FSK e PSK mas com características espectrais particulares, em que se verifica assimetria espectral em torno da freqüência central. Os sinais codificados apresentam envoltória constante, interessante para aplicação em canais não-lineares. Aspectos referentes à decodificação são comentados brevemente.

\section{Introdução}

Sayegh [1] propôs uma classe de esquemas de modulação codificada em uma e duas dimensões, controlados por códigos de bloco binários. Seu processo de construção multi-linha é creditado a Imai e Hirakawa [2], tendo sido também utilizado por Cusack [3] no projeto de esquemas combinando constelações QAM com códigos de Reed-Muller. A mesma técnica de construção será aqui adotada, estendendo-se os resultados obtidos a constelaçōes em três e quatro dimensões.

Seja C o conjunto de M sinais da constelação Q-dimensional adotada

$$
C=\left\{c_{0}(t), c_{1}(t), \ldots, c_{M-1}(t)\right\}
$$

cada qual associado a uma representação binária distinta

$$
b^{i}=\left\{\begin{array}{ccc}
(i) & \text { (i) } & \text { (i) } \\
b_{0}, & b_{1} & , \ldots, b_{m-1}
\end{array}\right\} \quad, i=0,1, \ldots, M-1
$$

A.C.F. Pessoa ê Pesquisador do CPqD - TELEBRÁs, Caixa Postai 1579, 13100, Campinas, SP. D.S. Arantes es Professor da Faculdade de Engenharia Elétrica da UNICAMP, Caixa Posta 6101, 13081, Campinas, SP. 
onde $\mathrm{m}$ é o logaritmo na base 2 de $\mathrm{M}$. Esta representação binária dos sinais segue 0 mapeamento por partição de conjuntos, introduzido por Ungerboeck [4].

O processo de codificação é descrito a seguir. Seja $\mathrm{V}$ uma matriz $\mathrm{mxN}$, binária, onde $\mathrm{N}$ é o comprimento das palavras codificadas.

$$
v=\left[\begin{array}{cccc}
v_{0,0} & v_{0,1} & \ldots & v_{0, N-1} \\
v_{1,0} & v_{1,1} & \ldots & v_{1, N-1} \\
\cdot & \cdot & & \cdot \\
\cdot & \cdot & & \cdot \\
v_{m-1,0} & v_{m-1,1} & \ldots & v_{m-1, N-1}
\end{array}\right]
$$

A cada linha $i$ de $V, i=0,1, \ldots, m-1$, é atribuída uma palavra de um código de bloco binário $\mathrm{B}_{\mathrm{i}}\left(\mathrm{N}, \mathrm{K}_{\mathrm{i}}, \mathrm{d}_{\mathrm{j}}\right)$, onde $\mathrm{K}_{\mathrm{i}}$ é o número de bits de informação deste código e $d_{i}$ sua distância mínima de Hamming. Cada coluna $j$ de $V, j=0,1, \ldots$, $\mathrm{N}-1$, é associada a um sinal $\mathrm{s}_{\mathrm{j}}(\mathrm{t})$ do conjunto $\mathrm{C}$, com representação vetorial $\left(v_{0, j}, v_{1, j}, \ldots, v_{m-1, j}\right)$. O bloco de sinais transmitido é dado por

$$
s(t)=\left(s_{0}(t), s_{1}(t), \ldots, s_{N-1}(t)\right)
$$

O número de bits de entrada do codificador, por bloco transmitido, é $\sum K_{i}$.

Se $R_{C}$ é a taxa de codificação, tem-se

$$
\sum_{i=0}^{m-1} K_{j}=m N R_{C}
$$

Para manter a taxa de sinalização de um sistema de referência não-codificado com $M / 2$ sinais, deve-se ter

$$
R_{C}=\frac{m-1}{m}
$$


e, conseqüentemente,

$$
\sum_{i=0}^{m-1} K_{i}=(m-1) N
$$

Pode-se demonstrar [5] que a distância Euclidiana mínima ao quadrado $D^{2}$ entre os vetores de sinais codificados é dada por

$$
D^{2}=\min \left[d_{0} \Delta_{0}^{2}, d_{1} \Delta_{1}^{2}, \ldots, d_{m-1} \Delta_{m-1}^{2}\right]
$$

onde

$$
\Delta_{j}=\min _{r \neq s}\left|c_{r}-c_{s}\right|^{2}
$$

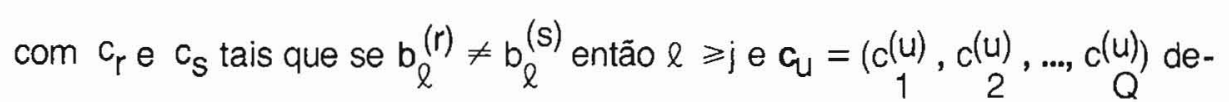
notando a representação vetorial do sinal $c_{U}(t)$, pertencente ao conjunto $C$ de dimensionalidade $\mathrm{Q}$. Tem-se assim

$$
c_{u}(t)=\sum_{q=1}^{Q} c_{q}^{(u)} \quad \lambda_{q} \cdot(t)
$$

onde

$$
\Lambda=\left\{\lambda_{1}(t), \quad \lambda_{2}(t), \quad \ldots, \quad \lambda_{Q}(l)\right\}
$$

é a base de sinais ortonormais adotada. Note-se ainda que em (8), os parâmetros $d_{j}, j=0,1, \ldots, m-1$, representam as distâncias mínimas de Hamming dos melhores códigos de bloco binários conhecidos [6] e [7] com comprimento $\mathrm{N}$ satisfazendo ( $\mathbf{7})$.

O ganho assintótico em relação ao sistema de referência utilizado é dado por

$$
G_{A}=10 \log _{10} \frac{D^{2}}{D_{\text {ref }}^{2}}
$$


onde $\mathrm{D}^{2}$ é a distância Euclidiana mínima ao quadrado entre os sinais do sistema de referência.

\section{Constelaçōes Espectralmente Assimétricas}

Os conjuntos de sinais tratados neste trabalho constituem essencialmente uma generalizaçāo da classe de constelações estudada por Markman [6], em que se explora uma melhor distribuição dos pontos no espaço de sinais. As constelações consideradas agrupam sinais da forma

$$
\begin{aligned}
& c_{i}(t)=\sqrt{\frac{2}{T}}\left[\frac{1}{\sqrt{1+\delta}} \cos \left(\omega_{C} t+\frac{\pi h t}{T}+\theta_{i}\right)+\frac{\sqrt{\delta}}{\sqrt{1+\delta}}\right. \\
& \left.\cos \left(\omega_{C} t-\frac{\pi h t}{T}+\phi_{i}\right)\right], \quad 0 \leqslant t<T, i=0,1,2, \ldots, M-1
\end{aligned}
$$

onde $\delta$, denominado fator de simetria, expressa o desbalanceamento espectral. Considerar-se-á $0 \leqslant \delta \leqslant 1$ pois neste intervalo ganhos podem ser obtidos em relação aos sistemas simétricos, que apresentam $\delta=1$. O estudo será concentrado nos casos em que h é inteiro pois sinais com envoltória constante, adequados a canais não-lineares, são obtidos nestas condições.

As funções ortonormais consideradas são

$$
\begin{array}{ll}
\lambda_{1}(t)=\sqrt{2 / T} \cos \left(\omega_{c} t+\pi h t / T\right) & , 0 \leqslant t<T \\
\lambda_{2}(t)=-\sqrt{2 / T} \operatorname{sen}\left(\omega_{c} t+\pi h t / T\right\rangle & , 0 \leqslant t<T \\
\lambda_{3}(t)=\sqrt{2 / T} \cos \left(\omega_{c} t+\pi h t / T\right) & , 0 \leqslant t<T \\
\lambda_{4}(t)=-\sqrt{2 / T} \operatorname{sen}\left(\omega_{c} t+\pi h t / T\right) & , 0 \leqslant t<T
\end{array}
$$

Desse modo, a representação vetorial dos sinais dados em (13) é do tipo 
$c_{i}=\left\langle\frac{1}{\sqrt{1+\delta}} \cos \theta_{i}, \frac{1}{\sqrt{1+\delta}} \operatorname{sen} \theta_{i}, \frac{\sqrt{\delta}}{\sqrt{1+\delta}} \cos \theta_{i}, \frac{\sqrt{\delta}}{\sqrt{1+\delta}} \operatorname{sen} \theta_{i}\right\rangle$

Estes esquemas em 3 e 4 dimensões serão denominados A2F/PxQ-PSK, $P \leqslant Q, P \times Q=M, c o m$

$$
\begin{aligned}
& \theta_{i} \in[0,2 \pi / Q, \ldots, 2 \pi(M-1) / Q] \\
& \phi_{i} \in[0,2 \pi / P, \ldots, 2 \pi(M-1) / P]
\end{aligned}
$$

Quando $\mathrm{P}=2$, o sistema é tri-dimensional; caso contrário, a constelação tem dimensionalidade igual a quatro.

As curvas de capacidade de canal para algumas constelações da classe acima são apresentadas nas figuras 1 a 3, para diversos valores de $\delta$, em função da razão sinal-ruído $\gamma=E / N_{0}$, onde $E$ é a energia média por sinal e $N_{0}$ é a densidade espectral unilateral de potência do ruído Gaussiano, branco e aditivo. As componentes do ruído em cada direção ortogonal apresentam média nula e variância $N_{0} / 2$.

Observa-se que a região ótima de $\delta$ em relação à capacidade de canal para o sistema A2F/2x4-PSK está em torno de $\delta=0,5$, com ganho teórico de aproximadamente $0,3 \mathrm{~dB}$ sobre o sistema espectralmente simétrico, $\operatorname{com} \delta=1$. Para o sistema A2F/2x8-PSK, aquela regiāo fica entre $\delta=0,25$ e $\delta=0,5$, com ganho teórico de aproximadamente $0,8 \mathrm{~dB}$. Para o sistema A2F/4x4-PSK, o esquema espectralmente simétrico é o mais indicado teoricamente.

Deve-se enfatizar que as limitações impostas pelo processo de construção multi-linha e pelo universo de códigos de bloco binários ótimos conhecidos introduzirão variações com relação aos resultados teóricos citados.

\section{Mapeamentos Binários e Relações de Distância}

Ao contrário dos esquemas convencionais, a classe de constelações apresentada na seção anterior possibilita a definição de vários bons mapeamentos binários por partição de conjuntos, com diferentes parâmetros de distâncias $\Delta_{0}$, $\Delta_{1}, \ldots \ldots, \Delta_{\mathrm{m}-1}$ que dependem do fator de simetria $\Delta$. O procedimento para determinação de mapeamentos interessantes e suas respectivas relações de distância é simples para constelações com poucos sinais e é exemplificado a seguir para a constelação A2F/2x4-PSK. Para este conjunto de sinais, as representações vetoriais dos mesmos são 


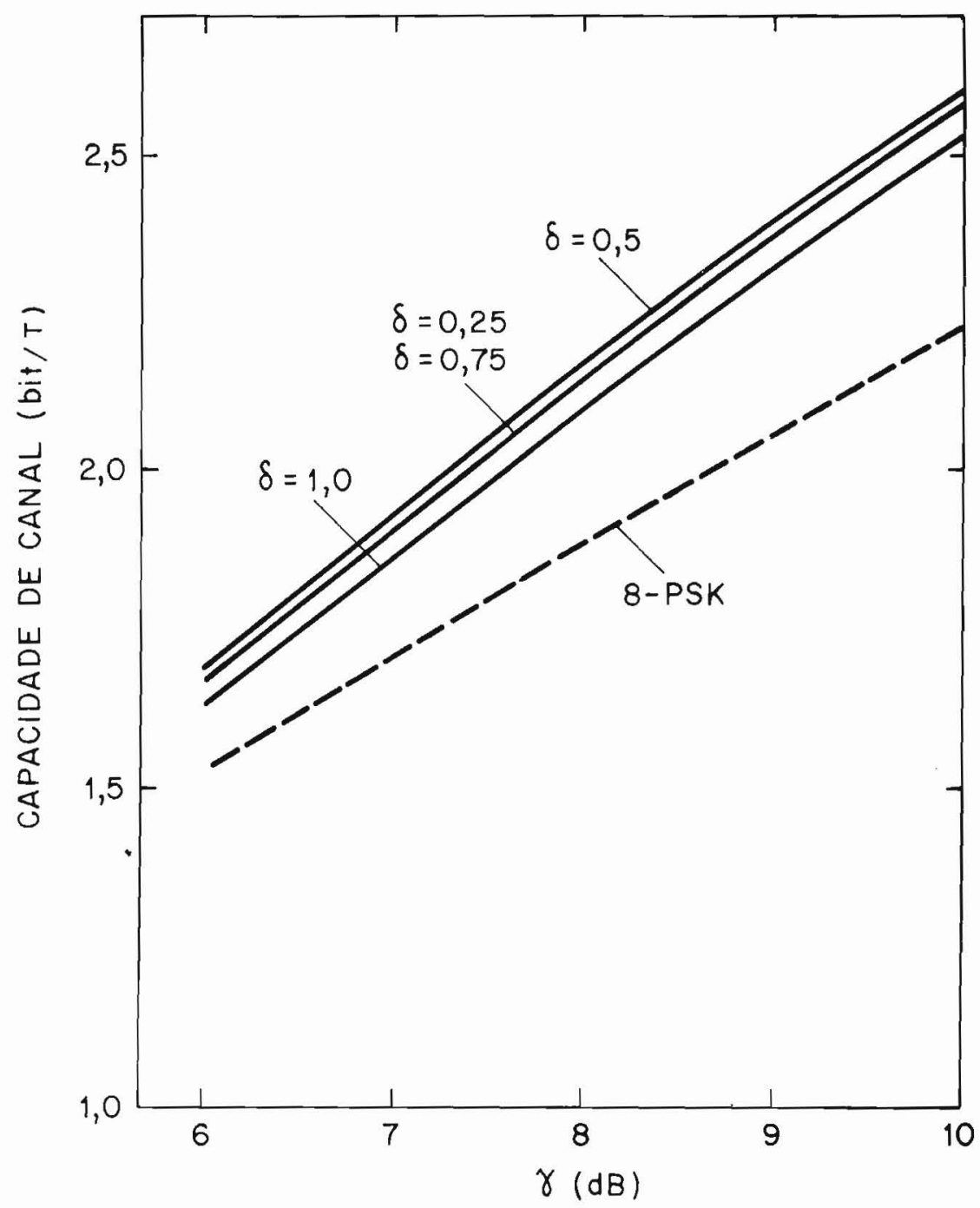

Figura 1. Capacidade de canal para o sistema A2F/2x4 - PSK. 


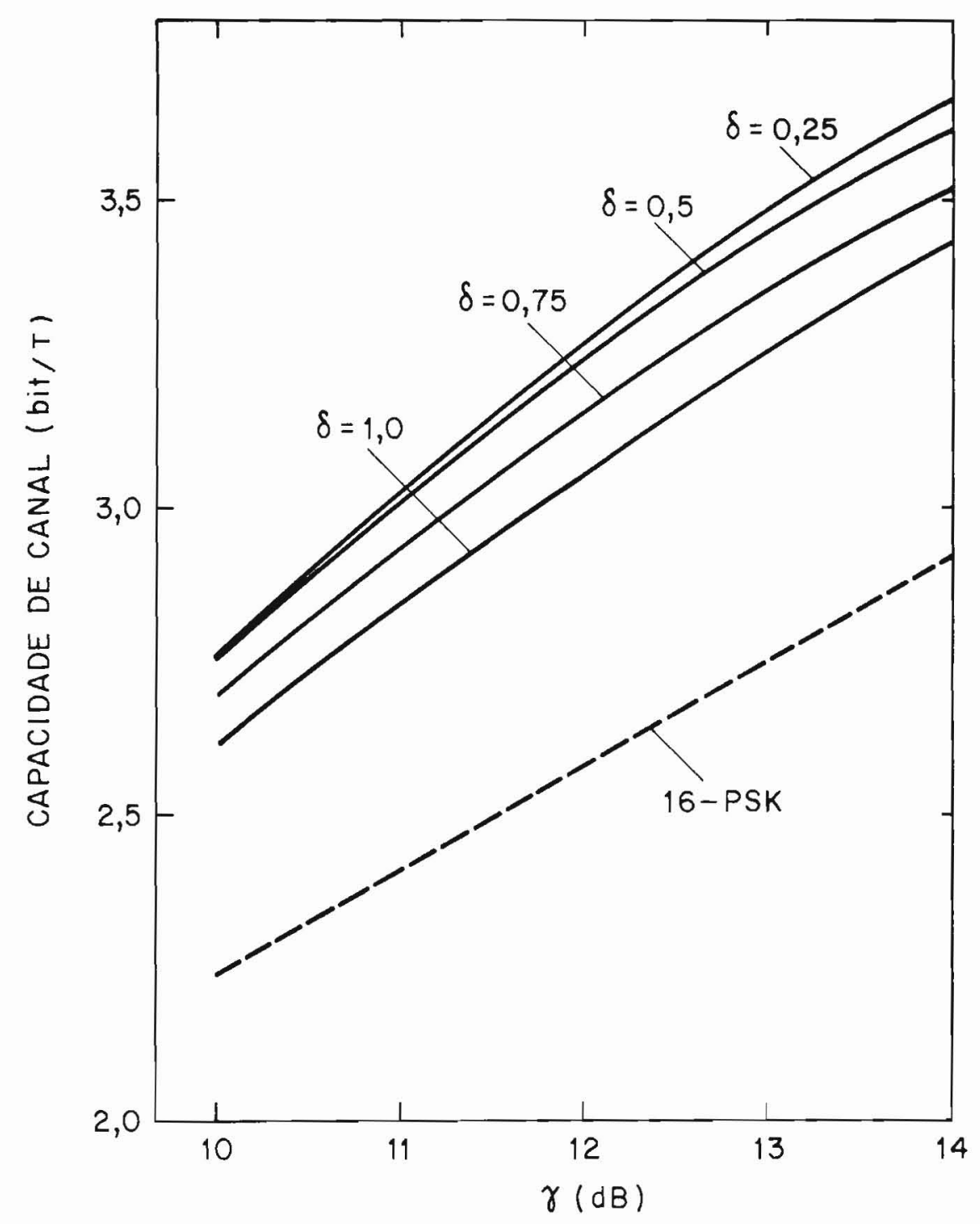

Figura 2. Capacidade de canal para o sistema A2F/2x8 - PSK. 


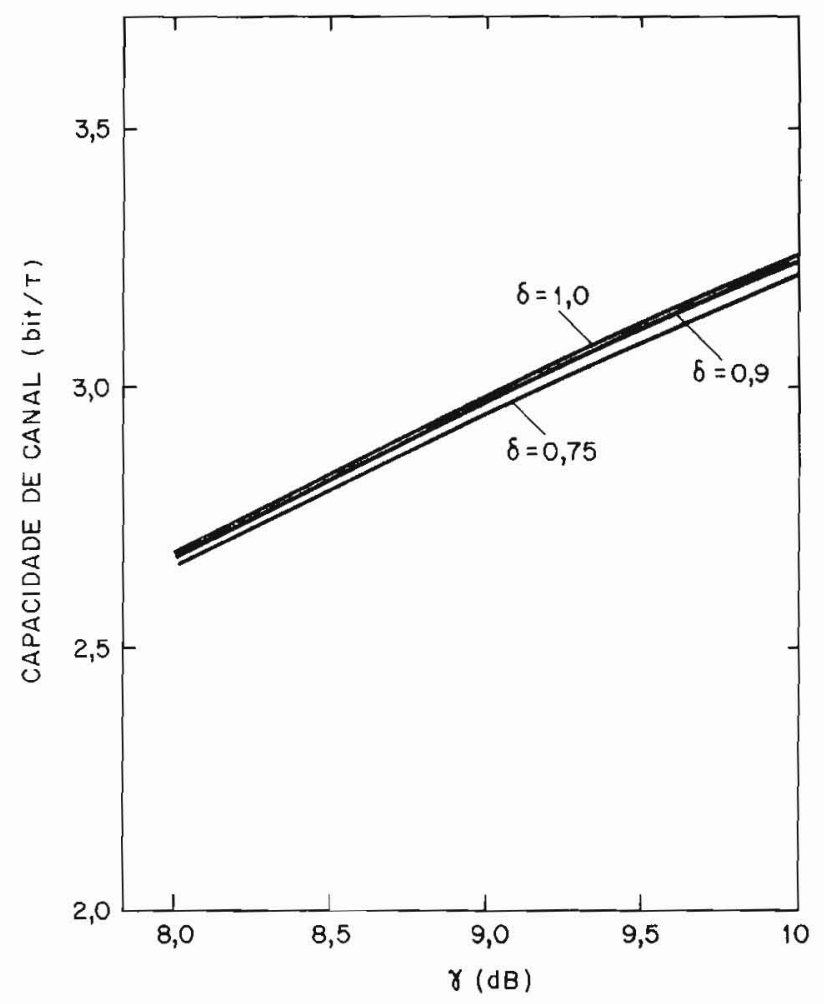

Figura 3. Capacidade de canal para o sistema A2F/4X4 - PSK.

$$
\begin{aligned}
& c_{0}=(1 / \sqrt{1+\delta}, 0, \sqrt{\delta} / \sqrt{1+\delta}, 0) \\
& c_{1}=(0,1 / \sqrt{1+\delta}, \sqrt{\delta} / \sqrt{1+\delta}, 0) \\
& c_{2}=(-1 / \sqrt{1+\delta}, 0, \sqrt{\delta} / \sqrt{1+\delta}, 0) \\
& c_{3}=(0,-1 / \sqrt{1+\delta}, \sqrt{\delta} / \sqrt{1+\delta}, 0) \\
& c_{4}=(1 / \sqrt{1+\delta}, 0,-\sqrt{\delta} / \sqrt{1+\delta}, 0) \\
& c_{5}=(0,1 / \sqrt{1+\delta},-\sqrt{\delta} / \sqrt{1+\delta}, 0) \\
& c_{6}=(-1 / \sqrt{1+\delta}, 0,-\sqrt{\delta} / \sqrt{1+\delta}, 0) \\
& c_{7}=(0,-1 / \sqrt{1+\delta},-\sqrt{\delta} / \sqrt{1+\delta}, 0)
\end{aligned}
$$


As distâncias Euclidianas ao quadrado entre os sinais desta constelação dependem do fator de simetria $\delta$ e estão relacionadas na Tabela 1, que utiliza os seguintes parâmetros

$$
\begin{aligned}
\varepsilon_{1} & =\frac{2}{1+\delta} \\
\varepsilon_{2} & =\frac{4}{1+\delta} \\
\varepsilon_{3} & =\frac{4 \delta}{1+\delta} \\
\varepsilon_{4} & =\frac{4 \delta+2}{1+\delta} \\
\varepsilon_{5} & =4
\end{aligned}
$$

\begin{tabular}{|c|c|c|c|c|c|c|c|c|c|}
\hline \multirow{2}{*}{\multicolumn{2}{|c|}{$\left|c_{i}-c_{j}\right|^{2}$}} & \multicolumn{8}{|c|}{ j } \\
\hline & & 0 & 1 & 2 & 3 & 4 & 5 & 6 & 7 \\
\hline \multirow{8}{*}{ i } & 0 & 0 & $\varepsilon_{1}$ & $\varepsilon_{2}$ & $\varepsilon_{1}$ & $\varepsilon_{3}$ & $\varepsilon_{4}$ & $\varepsilon_{5}$ & $\varepsilon_{4}$ \\
\hline & 1 & $\varepsilon_{1}$ & 0 & $\varepsilon_{1}$ & $\varepsilon_{2}$ & $\varepsilon_{4}$ & $\varepsilon_{3}$ & $\varepsilon_{4}$ & $\varepsilon_{5}$ \\
\hline & 2 & $\varepsilon_{2}$ & $\varepsilon_{1}$ & 0 & $\varepsilon_{1}$ & $\varepsilon_{5}$ & $\varepsilon_{4}$ & $\varepsilon_{3}$ & $\varepsilon_{4}$ \\
\hline & 3 & $\varepsilon_{1}$ & $\varepsilon_{2}$ & $\varepsilon_{1}$ & 0 & $\varepsilon_{4}$ & $\varepsilon_{5}$ & $\varepsilon_{4}$ & $\varepsilon_{3}$ \\
\hline & 4 & $\varepsilon_{3}$ & $\varepsilon_{4}$ & $\varepsilon_{5}$ & $\varepsilon_{4}$ & 0 & $\varepsilon_{1}$ & $\varepsilon_{2}$ & $\varepsilon_{1}$ \\
\hline & 5 & $\varepsilon_{4}$ & $\varepsilon_{3}$ & $\varepsilon_{4}$ & $\varepsilon_{5}$ & $\varepsilon_{1}$ & 0 & $\varepsilon_{1}$ & $\varepsilon_{2}$ \\
\hline & 6 & $\varepsilon_{5}$ & $\varepsilon_{4}$ & $\varepsilon_{3}$ & $\varepsilon_{4}$ & $\varepsilon_{2}$ & $\varepsilon_{1}$ & 0 & $\varepsilon_{1}$ \\
\hline & 7 & $\varepsilon_{4}$ & $\varepsilon_{5}$ & $\varepsilon_{4}$ & $\varepsilon_{3}$ & $\varepsilon_{1}$ & $\varepsilon_{2}$ & $\varepsilon_{1}$ & 0 \\
\hline
\end{tabular}

Tabela 1. Distância Euclidiana ao Quadrado entre os sinais da constelação A2F/2x4-PSK. 
As curvas de $\varepsilon_{1}$ a $\varepsilon_{5}$ em função de $\delta$ são apresentadas na Fig. 4. Verifica-se que

$$
\varepsilon_{3} \leqslant \varepsilon_{1} \leqslant \varepsilon_{4} \leqslant \varepsilon_{2} \leqslant \varepsilon_{5} \text {, para } 0 \leqslant \delta \leqslant 0,5
$$

e

$$
\varepsilon_{1} \leqslant \varepsilon_{3} \leqslant \varepsilon_{2} \leqslant \varepsilon_{4} \leqslant \varepsilon_{5} \text {, para } 0,5 \leqslant \delta \leqslant 1,0
$$

Esta propriedade auxilia a escolha de mapeamentos convenientes para cada intervalo de fator de simetria citado anteriormente.

A Tabela 2 apresenta os quatro mapeamentos relevantes para a constelação

\begin{tabular}{|c|c|c|c|c|}
\hline$\delta$ & \multicolumn{2}{|c|}{$0<\delta<0.5$} & \multicolumn{2}{|c|}{$0,5<\delta<1,0$} \\
\hline Mapeamento & 1 & 2 & 3 & 4 \\
\hline Sinal & \multicolumn{4}{|c|}{ Representação Binária $\left(\mathrm{b}_{0}^{(\mathrm{i})}, \mathrm{b}_{1}^{(\mathrm{i})}, \mathrm{b}_{2}^{(\mathrm{i})}\right)$} \\
\hline$c_{0}$ & 000 & 000 & 000 & 000 \\
\hline$c_{1}$ & 010 & 110 & 100 & 100 \\
\hline $\mathrm{C}_{2}$ & 101 & 001 & 011 & 010 \\
\hline$c_{3}$ & 111 & 111 & 111 & 110 \\
\hline$c_{4}$ & 100 & 100 & 010 & 111 \\
\hline$c_{5}$ & 110 & 010 & 110 & 001 \\
\hline$c_{6}$ & 001 & 101 & 001 & 101 \\
\hline$c_{7}$ & 011 & 011 & 101 & 011 \\
\hline$\Delta_{0}^{2}$ & $\varepsilon_{3}$ & $\varepsilon_{3}$ & $\varepsilon_{1}$ & $\varepsilon_{1}$ \\
\hline$\Delta_{1}^{2}$ & $\varepsilon_{1}$ & $\varepsilon_{4}$ & $\varepsilon_{3}$ & $\varepsilon_{2}$ \\
\hline$\Delta_{2}^{2}$ & $\varepsilon_{5}$ & $\varepsilon_{2}$ & $\varepsilon_{5}$ & $\varepsilon_{4}$ \\
\hline
\end{tabular}
considerada, sujeitos a intervalos de fator de simetria específicos.

Tabela 2: Mapeamentos binários para a constelação A2F/2x4-PSK. 


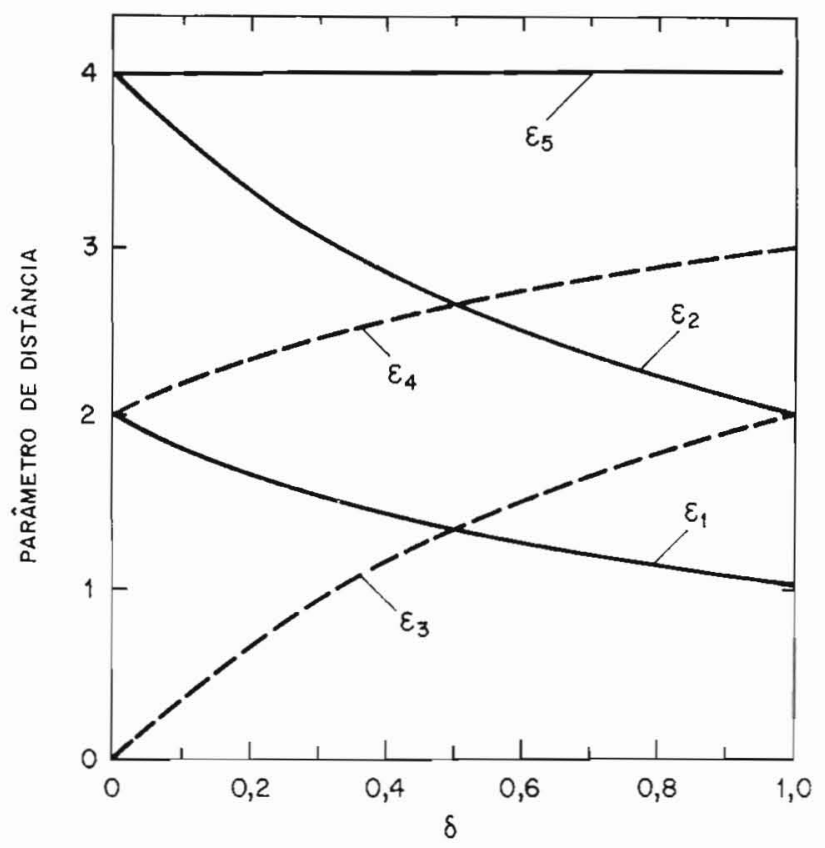

Figura 4. Parâmetros de distância da constelação A2F/2x4 - PSK versus o fator de simetria $\delta$.

\section{A Busca de Bons Códigos}

Seja $\mathrm{P}$ o conjunto dos $\mathrm{r}$ mapeamentos por partição de conjunto escolhidos,

$$
P=\left\{P_{1}, P_{2}, \ldots \ldots P_{r}\right\}
$$

O problema de otimização que objetiva maximizar a mínima distância Euclidiana ao quadrado entre os sinais codificados pode ser expresso como:

$$
\operatorname{máx}_{1 \leqslant i \leqslant r, 0 \leqslant \delta \leqslant 1} D^{2}\left(P_{i}, \delta\right)
$$

onde

$$
D^{2}\left(P_{i}, \delta\right)=\min \left[d_{0} \Delta_{C}^{2}\left(P_{i}, \delta\right), \ldots, d_{m-1} \Delta_{m-1}^{2}\left(P_{i}, \delta\right)\right]
$$


com $\Delta^{2}\left(P_{i}, \delta\right)$ expressando a dependência de $\Delta^{2}, j=0,1, \ldots \ldots, m-1$, com relação a $\delta$ e ao mapeamento $P_{i} \in P$.Conforme especificado anteriormente, os parâmetros $d_{j}, j=0,1, \ldots ., m-1$, representam distâncias mínimas de Hamming.

O esforço computacional necessário para busca exaustiva dos melhores esquemas, cada qual associado a um mapeamento por partição de conjuntos e a um fator de simetria específicos, é reduzido para os sistemas e comprimentos $(\mathrm{N} \leqslant 32)$ considerados. A Tabela 3 apresenta os melhores esquemas e os ganhos assintóticos obtidos para constelaçōes do tipo A2F/2×4-PSK considerando-se o sistema 4-PSK como referência. Para os valores de $N$ não citados, o mesmo ganho assintótico pode ser obtido com um código de menor comprimento.

\begin{tabular}{|c|c|c|r|r|r|r|r|r|r|c|}
\hline $\mathrm{N}$ & $\delta$ & Mapeamento & $\mathrm{D}^{2}$ & $\mathrm{G}_{\mathrm{A}}(\mathrm{dB})$ & $\mathrm{K}_{0}$ & $\mathrm{~K}_{1}$ & $\mathrm{~K}_{2}$ & $\mathrm{~d}_{0}$ & $\mathrm{~d}_{1}$ & $\mathrm{~d}_{2}$ \\
\hline 2 & $1 / 2$ & 3 ou 4 & 2,67 & 1,25 & 1 & 1 & 2 & 2 & 2 & 1 \\
3 & $3 / 4$ & 3 & 3,43 & 2,34 & 1 & 2 & 3 & 3 & 2 & 1 \\
4 & 1 & 3 & 4,00 & 3,01 & 1 & 3 & 4 & 4 & 2 & 1 \\
6 & $1 / 2$ & 4 & 5,33 & 4,26 & 2 & 5 & 5 & 4 & 2 & 2 \\
13 & $3 / 4$ & 4 & 5,71 & 4,56 & 5 & 9 & 12 & 5 & 3 & 2 \\
14 & $5 / 8$ & 3 & 6,15 & 4,88 & 6 & 9 & 13 & 5 & 4 & 2 \\
15 & $3 / 4$ & 3 & 6,86 & 5,35 & 6 & 10 & 14 & 6 & 4 & 2 \\
18 & $7 / 8$ & 3 & 7,47 & 5,72 & 7 & 12 & 17 & 7 & 4 & 2 \\
19 & 1 & 3 & 8,00 & 6,02 & 7 & 13 & 18 & 8 & 4 & 2 \\
22 & $2 / 3$ & 4 & 8,40 & 6,23 & 11 & 16 & 17 & 7 & 4 & 3 \\
23 & $1 / 2$ & 4 & 9,33 & 6,69 & 12 & 17 & 17 & 7 & 4 & 4 \\
24 & $1 / 2$ & 4 & 10,67 & 7,27 & 12 & 18 & 18 & 8 & 4 & 4 \\
\hline
\end{tabular}

Tabela 3. Constelação A2F/2x4-PSK: melhores esquemas; sisteme de referência 4-PSK $\left(D_{\text {ref }}^{2}=2\right)$. 
$\mathrm{Na}$ Fig. 5, o esquema A2F/2x4-PSK é comparado com o sistema espectralmente simétrico associado, no que diz respeito ao ganho assintótico em função de N, o comprimento do código.

As tabelas 4 e 6 expõem os melhores esquemas obtidos com as constelações A2F/2x8-PSK e A2F/4x4-PSK. As tabelas 5 e 7 apresentam os mapeamentos selecionados no processo de otimização. Curvas de comparação destes esquemas com os que utilizam constelações espectralmente simétricas aparecem nas figuras 6 e 7 .

Os resultados obtidos refletem o comportamento sugerido pelas curvas de capacidade de canal apresentadas nas figuras 1 até 3 , tanto no que se refere aos ganhos obtidos com relação às constelações espectralmente simétricas quanto às regiões favoráveis do fator de simetria, com algumas variações impostas pelo processo de codificação e disponibilidade de bons códigos binários.

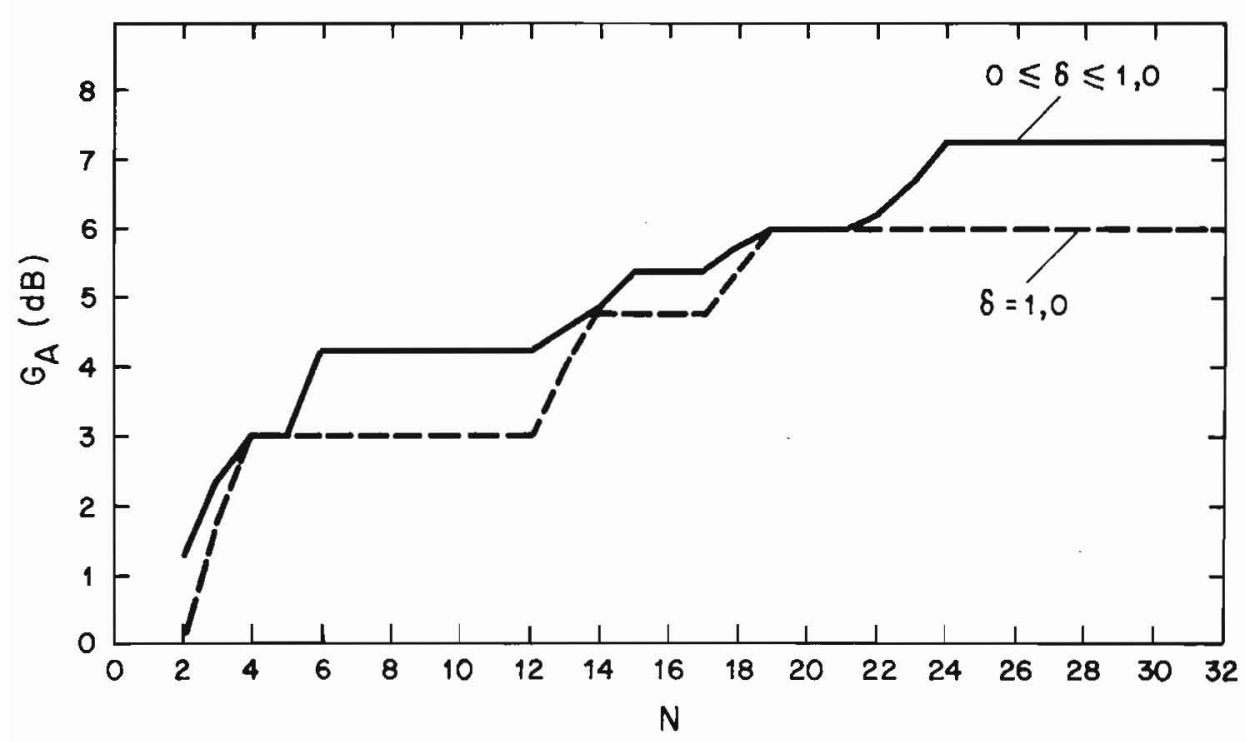

Figura 5. Ganho assintótico versus comprimento do código: comparaçāo entre o sistema A2F/2x4 - PSK ótimo e o sistema espectralmente simétrico correspondente. 


\begin{tabular}{|c|c|c|c|c|c|c|c|c|c|c|c|c|}
\hline $\mathrm{N}$ & $\delta$ & Mapeamento & $D^{2}$ & $G_{A}(d B)$ & $\mathrm{K}_{0}$ & $\mathrm{~K}_{1}$ & $\mathrm{~K}_{2}$ & $\mathrm{~K}_{3}$ & $d_{0}$ & $d_{1}$ & $d_{2}$ & $d_{3}$ \\
\hline 2 & $\frac{2-\sqrt{2}}{4}$ & 1 & 1,02 & 2,42 & 1 & 1 & 2 & 2 & 2 & 2 & 1 & 1 \\
\hline 3 & $\frac{2-\sqrt{2}}{4}$ & 1 & 1,53 & 4,18 & 1 & 2 & 3 & 3 & 3 & 2 & 1 & 1 \\
\hline 4 & $\frac{2-\sqrt{2}}{2}$ & 2 & 1,81 & 4,91 & 1 & 3 & 4 & 4 & 4 & 2 & 1 & 1 \\
\hline 5 & $\frac{10-5 \sqrt{2}}{8}$ & 2 & 2,14 & 5,63 & 1 & 4 & 5 & 5 & 5 & 2 & 1 & 1 \\
\hline 6 & $\frac{6-3 \sqrt{2}}{4}$ & 2 & 2,44 & 6,20 & 1 & 5 & 6 & 6 & 6 & 2 & 1 & 1 \\
\hline 7 & $1 / 2$ & 2 & 2,67 & 6,58 & 1 & 6 & 7 & 7 & 7 & 2 & 1 & 1 \\
\hline 11 & $\frac{\sqrt{2}}{4}$ & 3 & 2,96 & 7,03 & 2 & 10 & 10 & 11 & 7 & 2 & 2 & 1 \\
\hline 14 & $\frac{2-\sqrt{2}}{4}$ & 1 & 3,07 & 7,19 & 5 & 10 & 13 & 14 & 6 & 3 & 2 & 1 \\
\hline 15 & $\frac{3 \sqrt{2}-2}{12}$ & 1 & 3,37 & 7,60 & 5 & 11 & 14 & 15 & 7 & 3 & 2 & 1 \\
\hline 18 & $1 / 7$ & 1 & 3,50 & 7,76 & 7 & 12 & 17 & 18 & 7 & 4 & 2 & 1 \\
\hline 20 & $\frac{2-\sqrt{2}}{2}$ & 2 & 3,62 & 7,92 & 8 & 14 & 19 & 19 & 8 & 4 & 2 & 2 \\
\hline 23 & $\frac{4-2 \sqrt{2}}{5}$ & 2 & 3,80 & 8,12 & 11 & 14 & 22 & 22 & 8 & 5 & 2 & 2 \\
\hline 24 & $\frac{2-\sqrt{2}}{4}$ & 1 & 4,09 & 8,44 & 12 & 18 & 19 & 23 & 8 & 4 & 3 & 2 \\
\hline
\end{tabular}

Tabela 4. Constelação A2F/2x8-PSK: melhores esquemas; sistema de referência $8-P S K\left(D^{2}\right.$ ref $\left.=2-\sqrt{2}\right)$. 


\begin{tabular}{|c|c|c|c|c|c|c|}
\hline \multicolumn{4}{|c|}{$\delta$} & $0 \leqslant \delta \leqslant \frac{\sqrt{2}}{4}$ & $\frac{2-\sqrt{2}}{4} \leqslant \delta \leqslant 1 / 2$ & $\frac{\sqrt{2}}{4}<\delta \leqslant 1 / 2$ \\
\hline \multicolumn{4}{|c|}{ Mapeamento } & 1 & 2 & 3 \\
\hline Sinal & $c_{1}{ }^{(i)}$ & $c_{2}^{(i)}$ & $c_{3}{ }^{(i)}$ & \multicolumn{3}{|c|}{ Representação binária $\left(b_{0}^{(i)}, \ldots, b_{3}{ }^{(i)}\right)$} \\
\hline C & $\gamma_{1}$ & 0 & $\gamma_{3}$ & 0000 & 0000 & 0000 \\
\hline$c_{1}$ & $\gamma_{2}$ & $\gamma_{2}$ & $\gamma_{3}$ & 1100 & 1000 & 1000 \\
\hline $\mathrm{c}_{2}$ & 0 & $\gamma_{1}$ & $\gamma_{3}$ & 0010 & 0110 & 0100 \\
\hline$c_{3}$ & $-\gamma_{2}$ & $\gamma_{2}$ & $\gamma_{3}$ & 1110 & 1110 & 1100 \\
\hline $\mathrm{C}_{4}$ & $-\gamma_{1}$ & 0 & $\gamma_{3}$ & 0001 & 0001 & 0011 \\
\hline$c_{5}$ & $-\gamma_{2}$ & $-\gamma_{2}$ & $\gamma_{3}$ & 1101 & 1001 & 1011 \\
\hline $\mathrm{c}_{6}$ & 0 & $-\gamma_{1}$ & $\gamma_{2}$ & 0011 & 0111 & 0111 \\
\hline$c_{7}$ & $\gamma_{2}$ & $-\gamma_{2}$ & $\gamma_{3}$ & 1111 & 1111 & 1111 \\
\hline$c_{8}$ & $\gamma_{1}$ & 0 & $-\gamma_{3}$ & 1000 & 0100 & 1010 \\
\hline $\mathrm{c}_{9}$ & $\gamma_{2}$ & $\gamma_{2}$ & $-\gamma_{3}$ & 0100 & 1100 & 0010 \\
\hline$c_{10}$ & 0 & $\gamma_{1}$ & $-\gamma_{3}$ & 1010 & 0010 & 1110 \\
\hline$c_{11}$ & $-\gamma_{2}$ & $\gamma_{2}$ & $-\gamma_{3}$ & 0110 & 1010 & 0110 \\
\hline$c_{12}$ & $-\gamma_{1}$ & 0 & $-\gamma_{3}$ & 1001 & 0101 & 1001 \\
\hline$c_{13}$ & $-\gamma_{2}$ & $-\gamma_{2}$ & $-\gamma_{3}$ & 0101 & 1101 & 0001 \\
\hline$c_{14}$ & 0 & $-\gamma_{1}$ & $-\gamma_{3}$ & 1011 & 0011 & 1101 \\
\hline$C_{15}$ & $\gamma_{2}$ & $-\gamma_{2}$ & $-\gamma_{3}$ & 0111 & 1011 & 0101 \\
\hline
\end{tabular}

Tabela 5. Mapeamentos para a constelação $A 2 F / 2 \times 8$ - PSK:

$$
\gamma_{1}=\frac{1}{\sqrt{1+\delta}}, \quad \gamma_{2}=\frac{\sqrt{2}}{2 \sqrt{1+\delta}}, \quad \gamma_{3}=\frac{\sqrt{\delta}}{\sqrt{1+\delta}}
$$




\begin{tabular}{|c|c|c|c|c|c|c|c|c|c|c|c|c|}
\hline $\mathrm{N}$ & $\delta$ & Mapeamento & $\mathrm{D}^{2}$ & $\mathrm{G}_{A}(\mathrm{~dB})$ & $\mathrm{K}_{\mathrm{O}}$ & $\mathrm{K}_{1}$ & $\mathrm{~K}_{2}$ & $\mathrm{~K}_{3}$ & $\mathrm{~d}_{\mathrm{O}}$ & $\mathrm{d}_{1}$ & $\mathrm{~d}_{2}$ & $\mathrm{~d}_{3}$ \\
\hline 2 & 1 & 1 ou 2 & 2,00 & 5,33 & 1 & 1 & 2 & 2 & 2 & 2 & 1 & 1 \\
3 & $2 / 3$ & 2 & 2,40 & 6,12 & 1 & 2 & 3 & 3 & 3 & 2 & 1 & 1 \\
4 & $1 / 2$ & 2 & 2,67 & 6,58 & 1 & 3 & 4 & 4 & 4 & 2 & 1 & 1 \\
5 & 1 & 1 & 3,00 & 7,09 & 2 & 4 & 4 & 5 & 3 & 2 & 2 & 1 \\
6 & 1 & 1 & 4,00 & 8,34 & 2 & 5 & 5 & 6 & 4 & 2 & 2 & 1 \\
15 & $4 / 5$ & 2 & 4,44 & 8,80 & 7 & 10 & 14 & 14 & 5 & 4 & 2 & 2 \\
16 & $2 / 3$ & 2 & 4,80 & 9,13 & 7 & 11 & 15 & 15 & 6 & 4 & 2 & 2 \\
19 & $4 / 7$ & 2 & 5,09 & 9,39 & 8 & 13 & 18 & 18 & 7 & 4 & 2 & 2 \\
20 & $1 / 2$ & 2 & 5,33 & 9,59 & 8 & 14 & 19 & 19 & 8 & 4 & 2 & 2 \\
21 & 1 & 1 & 6,00 & 10,10 & 11 & 16 & 16 & 20 & 6 & 3 & 3 & 2 \\
26 & 1 & 1 & 7,00 & 10,77 & 13 & 20 & 20 & 25 & 7 & 4 & 4 & 2 \\
27 & 1 & 1 & 8,00 & 11,35 & 13 & 21 & 21 & 26 & 8 & 4 & 4 & 2 \\
\hline
\end{tabular}

Tabela 6. Constelação A2F/4x4-PSK: melhores esquemas; sistema de referência 8-PSK $\left(D^{2}\right.$ ref $\left.=2-\sqrt{2}\right)$ 


\begin{tabular}{|c|c|c|c|c|c|c|}
\hline \multicolumn{5}{|c|}{$\delta$} & \multicolumn{2}{|c|}{$0,5 \leqslant \delta \leqslant 1,0$} \\
\hline \multicolumn{5}{|c|}{ Mapeamento } & 1 & 2 \\
\hline Sinal & $c_{1}^{(i)}$ & $c_{2}^{(i)}$ & $c_{3}^{(i)}$ & $\mathrm{c}_{4}^{(i)}$ & \multicolumn{2}{|c|}{ Representação binária $\left(b_{0}^{(i)}, \ldots, b_{3}^{(i)}\right)$} \\
\hline$c_{0}$ & $\gamma_{1}$ & 0 & $\gamma_{2}$ & 0 & 0000 & 0000 \\
\hline$c_{1}$ & 0 & $\gamma_{1}$ & $\gamma_{2}$ & 0 & 1110 & 0100 \\
\hline $\mathrm{c}_{2}$ & $-\gamma_{1}$ & 0 & $\gamma_{2}$ & 0 & 0101 & 0010 \\
\hline$c_{3}$ & 0 & $-\gamma_{1}$ & $\gamma_{2}$ & 0 & 1011 & 0110 \\
\hline $\mathrm{C}_{4}$ & $\gamma_{1}$ & 0 & 0 & $\gamma_{2}$ & 1000 & 1000 \\
\hline$c_{5}$ & 0 & $\gamma_{1}$ & 0 & $\gamma_{2}$ & 0010 & 1100 \\
\hline$c_{6}$ & $-\gamma_{1}$ & 0 & 0 & $\gamma_{2}$ & 1101 & 1010 \\
\hline$c_{7}$ & 0 & $-\gamma_{1}$ & 0 & $\gamma_{2}$ & 0111 & 1110 \\
\hline$c_{8}$ & $\gamma_{1}$ & 0 & $-\gamma_{2}$ & 0 & 0100 & 0101 \\
\hline $\mathrm{C}_{9}$ & 0 & $\gamma_{1}$ & $-\gamma_{2}$ & 0 & 1010 & 0001 \\
\hline$c_{10}$ & $-\gamma_{1}$ & 0 & $-\gamma_{2}$ & 0 & 0001 & 0111 \\
\hline$c_{11}$ & 0 & $-\gamma_{1}$ & $-\gamma_{2}$ & 0 & 1111 & 0011 \\
\hline$c_{12}$ & $\gamma_{1}$ & 0 & 0 & $-\gamma_{2}$ & 1100 & 1101 \\
\hline$c_{13}$ & 0 & $\gamma_{1}$ & 0 & $-\gamma_{2}$ & 0110 & 1001 \\
\hline$c_{14}$. & $-\gamma_{1}$ & 0 & 0 & $-\gamma_{2}$ & 1001 & 1111 \\
\hline$c_{15}$ & 0 & $-\gamma_{1}$ & 0 & $-\gamma_{2}$ & 0011 & 1011 \\
\hline
\end{tabular}

Tabela 7. Mapeamentos para a constelação A2F/4x4-PSK:

$$
\gamma_{1}=\frac{1}{\sqrt{1+\delta}} \quad, \quad \gamma_{2}=\frac{\sqrt{2}}{2 \sqrt{1+\delta}}
$$




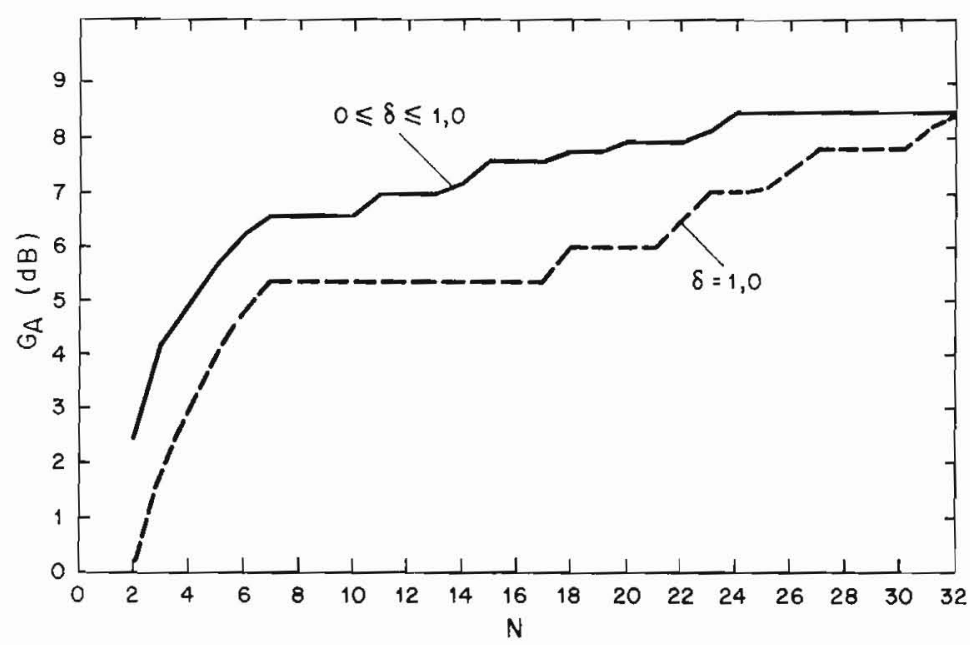

Figura 6. Ganho assintótico versus comprimento do código: comparação entre o sistema A2F/2x8 - PSK ótimo e o sistema espectralmente simétrico correspondente.

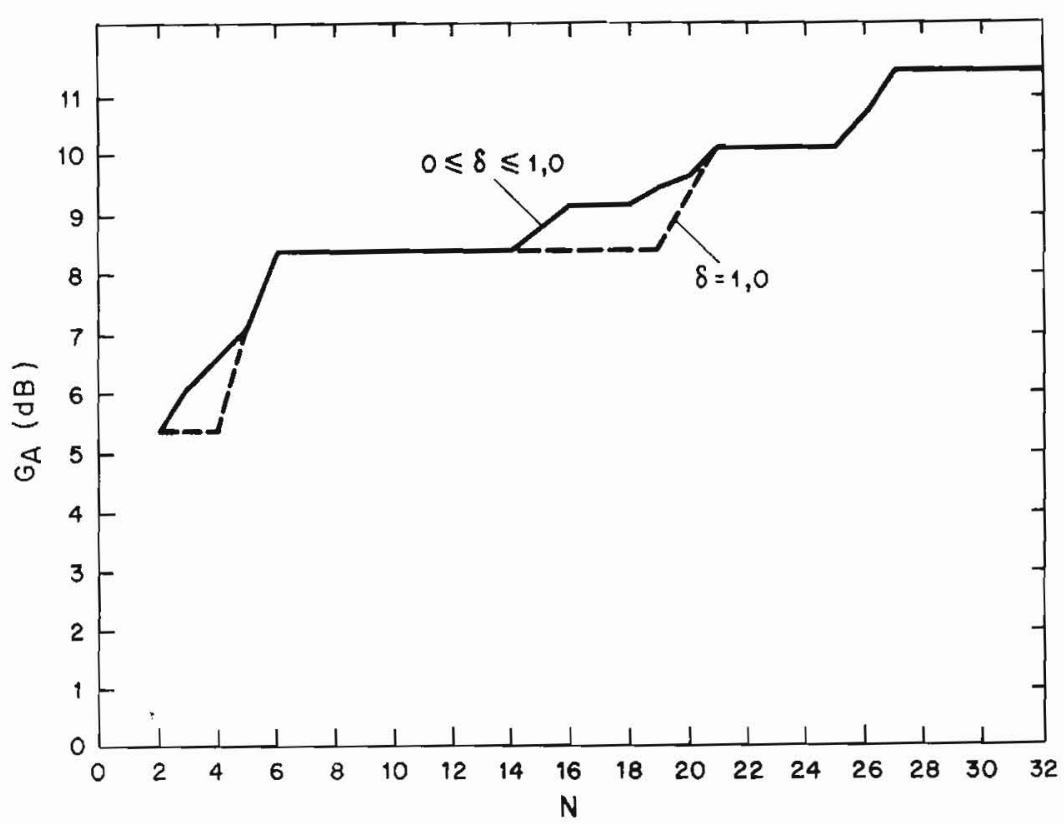

Figura 7. Ganho assintótico versus comprimento do código: comparação entre o sistema A2F/4x4 - PSK ótimo e o sistema espectralmente simétrico correspondente. 


\section{Caracteristicas Espectrais e Decodificação}

Nesta seção, os resultados alcançados em [9] são utilizados para determinar a densidade espectral de potência dos sinais dos sistemas de modulação codificada espectralmente assimétricos tratados. Quando os símbolos de entrada do codificador são estatisticamente independentes e equiprováveis, o espectro $R(f)$ destes sinais em banda básia é dado por

$$
\begin{aligned}
R(f)= & \frac{1}{T} \sum_{r=1}^{Q} Z_{r}\left|\Lambda_{r}(f)\right|^{2}+\frac{2}{N T} \sum_{n=1}^{N-1} \cos (2 \pi f n T) \sum_{r=1}^{Q}\left|\Lambda_{r}(f)\right|^{2} . \\
& \underset{i=0}{N-n-1} Z_{r r}^{(Y(i, i+n))}
\end{aligned}
$$

onde

$Z_{r}=\frac{1}{M} \sum_{k=0}^{M-1}\left[c_{r}(k)\right]^{2}$

$Z_{r r}^{(i)}=\frac{2^{i}}{M^{2}} \sum_{k=0}^{M-1} \sum_{k^{\prime}=0}^{M-1} \Delta^{(i)}\left(k, k^{\prime}\right) c_{r}{ }^{(k)} c_{s}{ }^{\left(k^{\prime}\right)}$

$$
\Delta^{(i)}\left(k, k^{\prime}\right)=\left\{\begin{array}{l}
1, \text { se } b_{j}(k)=b_{j}\left(k^{\prime}\right), j=0,1, \ldots, i-1 \\
0, \text { caso contrário }
\end{array}\right.
$$

$Y(j, j)$ é o número de pares de elementos da matriz-código pertencentes às colunas j e j' distintas e à mesma linha, sujeitos a dependência total; e $\Lambda_{r}(f)$ é a transformada de Fourier da representação em banda básica de $\lambda_{r}(t)$.

Quando, além das condições anteriores, nenhum par de elementos da matrizcódigo está sujeito a dependência total, a densidade espectral de potência dos sinais codificados, em banda básica, é dada por

$R(f)=\frac{2}{1+\delta} S a^{2}\left[\pi t\left(f+\frac{h}{2 T}\right)\right]+\frac{2 \delta}{1+\delta} S a^{2}\left[\pi t\left(f-\frac{h}{2 T}\right)\right]$

onde $S a(x)=\sin (x) / x$. 
Para $\delta<1$, existe uma maior concentração de potência em torno da freqüência -h/2T. Conseqüentemente, ocorre uma redução de faixa em relação aos sistemas espectralmente simétricos.

Sayegh [1] apresentou um esquema sub-ótimo para decodificação dos sistemas de modulação codificada que utilizam construções multi-linha. Trata-se de um decodificador etapa-por-etapa, em que as linhas são sucessivamente decodificadas. A complexidade destas etapas pode ser reduzida transformando-as em problemas unidimensionais e adotando o algoritmo de Chase para realizar decodificação simplificada com desempenho assintoticamente ótimo [10].

\section{Conclusões}

Neste trabalho, foi apresentada uma classe de sistemas de modulação codificada de bloco, em três e quatro dimensōes, espectralmente assimétricos, com ganhos de até 2,5 dB sobre os sistemas com espectros simétricos para os casos tratados (até 16 sinais). A densidade espectral de potência dos sinais codificados e o problema de decodificação são comentados brevemente e referências que tratam especificamente destes tópicos são apresentadas no texto. A extensão para constelações com maior número de sinais é simples e o problema de otimização menos complexo que o que seria demandado com a utilização de códigos convolucionais.

\section{Referências}

[1] S.L. Sayegh, "A Class of Optimum Block Codes in Signal Space", IEEE Transactions on Communications, vol. COM-34, n 10 , Outubro 1986, pp. 1043-1045.

[2] H. Imai e S. Hirakawa, "A New Multilevel Coding Method Using Error-Correcting Codes", IEEE Transactions on Information Theory, vol. IT-23, nํ 3 , Maio 1977, pp. 371-377.

[3] E.L. Cușack, "Error Control Codes for QAM Signalling", Electronics Letters, vol. 20, 1984, pp. 62-63.

[4] G. Ungerboeck, "Channel Coding with Multilevel/Phase Signals", IEEE Transactions on Information Theory, vol. IT-28, no 1, Janeiro 1982, pp. 5567. 
[5] A.C.F. Pessoa, "Esquemas de Modulação Codificada de Bloco com Construção Multi-Linha", Tese de Mestrado, Universidade Estadual de Campinas, SP, Junho, 1990.

[6] F.J. Macwilliams e N.J.A. Sloane, "The Theory of Error-Correcting Codes", North-Holland, 1977.

[7] T. Verhoeff, "An Updated Table of Minimum-Distance Eounds for Binary Linear Codes", IEEE Transactions on Information Theory, vol. IT-33, $n=5$, Setembro 1987, pp. 665-680.

[8] I. Markman, "Esquemas de Modulação Codificada em Três e Quatro Dimensões", Tese de Mestrado, Universidade Estadual de Campinas, SP, Junho, 1987.

[9] A.C.F. Pessoa e D.S. Arantes, "Cálculo de Espectro de Sinais de uma Classe de Sistemas de Modulação Codificada de Bloco", Anais do 6ㅇ Simpósio Brasileiro de Telecomunicaçōes, Campina Grande, PB, Setembro 1988, pp. 105-109.

[10] A.C.F. Pessoa e D.S. Arantes, "Decoding of Block Coded Signals Using the Chase Algorithm", apresentado no International Symposium on Information and Coding Theory, ISICT/87, Campinas, Julho, 1987.

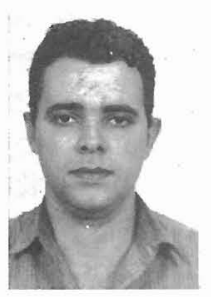

ANTONIO CLÁUDIO FRANÇA PESSOA nasceu no Rio de Janeiro em 23 de outubro de 1963. Formou-se em Engenharia Elétrica pela Universidade Federal da Paraíba/Campina Grande em 1985 e obteve o grau de Mestre em Ciências em Engenharia Elétrica pela UNICAMP em 1990. Desde 1987 trabalha no Centro de Pesquisa e Desenvolvimento (CPqD) da TELEBRÁS, onde atuou na especificação do sistema de proteção contra erros de canal do projeto RADIO DIGITAL 64-QAM e participa da equipe responsåvel pelo desenvolvimento de um equipamento para transmissão digital de TV comercial a $34 \mathrm{Mbit} / \mathrm{s}$. Suas áreas de interesse são Processamento Digital de Imagens, Códigos Corretores de Erros, Criptografia e Teoria da Informação.

DALTON SOARES ARANTES, fotografia e biografia podem ser encontradas neste número, p. 2. 